\title{
An ultrastructural study of the surface and attachment structures of Paradiplozoon homoion (Bychowsky \& Nagibina, 1959) (Monogenea: Diplozoidae)
}

\author{
Veronika Konstanzová1*, Božena Koubková1, Martin Kašný1,2, Jana llgová1, Ewa Dzika³ and Milan Gelnar
}

\begin{abstract}
Background: Species of Diplozoon Palombi, 1949 (Monogenea: Diplozoidae) are blood-feeding ectoparasites mainly parasitising the gills of cyprinid fishes. Although these parasites have been the subject of numerous taxonomic, phylogenetic and ecological studies, the ultrastructure of the surface and haptor attachment structures remains almost unknown. In this study, we used transmission electron microscopy to examine the ultrastructure of attachment clamps and neodermal surface of Paradiplozoon homoion (Bychowsky \& Nagibina, 1959), family Diplozoidae Palombi, 1949, thereby broadening our knowledge of platyhelminth biology.

Results: The hindbody surface of $P$. homoion is distinctly ridged, each ridge being supported by several muscle fibers and equipped with scales on the surface plasma membrane. Such structures have not been recorded previously in species of the family Diplozoidae. Comparisons of the surface structure of different body parts revealed slight differences in the thickness and number of organelles. Each of the clamps has a flattened bowl-like structure composed of sclerites, movable skeletal-like structures that are anchored by robust, radially oriented muscle bundles. The base of the posterior median plate sclerites is equipped with glandular cells possessing secretory vesicles.

Conclusion: This study brings detailed ultrastructural data for the surface and haptoral attachment clamps of P. homoion and provides new insights into the ultrastructure of Diplozoidae. Glandular cells at the base of the attachment clamps responsible for sclerite development in diplozoid species were observed for the first time. Our findings support the hypothesis that the structure of particular neodermal compartments is similar within the Platyhelminthes. On the other hand, the diplozoid glandular system and the mechanism of sclerite development clearly merits further attention.
\end{abstract}

Keywords: Paradiplozoon homoion, Ultrastructure, Neodermis, Tegument, Attachment clamps

\section{Background}

Species of Diplozoon Nordmann, 1832 are blood-feeding ectoparasites mainly parasitising the gills of cyprinid fishes, where they can cause mechanical damage to the gill filaments, initiating the development of secondary infections (bacterial, mycotic) and anemia [1, 2]. During the diplozoon life-cycle, two larvae (diporpa) pair and subsequently fuse

\footnotetext{
* Correspondence: 184753@mail.muni.cz

'Department of Botany and Zoology, Faculty of Science, Masaryk University, Kotlářská 2, 61137 Brno, Czech Republic

Full list of author information is available at the end of the article
}

permanently, producing the typical X-shaped diplozoon body arrangement, a characteristic unique to the Diplozoidae Palombi, 1949 [3]. Diplozoon spp. have developed a number of adaptations for successful attachment to gills, including central hooks and clamps located on the posterior adhesive organ, the haptor. The neodermis (tegument) in these species is also associated with a number of surface structures that play an important role in the ectoparasitic life-style. The neodermis has been investigated at the ultrastructural level in other monogenean species, including Gyrodactylus sp. [4, 5], Entobdella soleae, Acanthocotyle 
elegans [6], Diclidophora merlangi [7], Rajonchocotyle emarginata, Plectanocotyle gurnardi [8], Diplectanum aequans [9], Allodiscocotyla diacanthi [10], and Metamicrocotyla macracantha [11]. Studies on species within the Diplozoidae, however, are missing. In general, the external surface of monogeneans is a syncytium formed by neodermal (tegumental) cells. The distal cytoplasm is connected via cytoplasmic junctions with the proximal cytoplasm of cell bodies located in the subsurface parenchyma [7, 12-14].

An important part of the diplozoid monogenean body is the complex of posterior attachment structures on each opisthaptor, comprising a pair of central hooks and four pairs of clamps $[15,16]$. These allow the parasite to attach to the gills using an extrinsic muscle/tendon system associated with a median J-shaped sclerite [17]. The clamps comprise a posterior and anterior jaw, joined with a median plate by anterior and posterior joining sclerites $[18,19]$. These clamps are morphologically distinct and can be used for species determination [19-22]. While the ultrastructure of heteronchoinean clamps has been investigated previously [23-28], ultrastructural data for diplozoids are presently missing. In general, the clamp wall surrounding the inner sclerites is formed of a specialized muscle complex with radially oriented muscle fibers [22]. Chemical analysis has shown that heteronchoinean sclerites, including those in Diplozoon paradoxum, consist of scleroproteins [29-32].

The main aim of this study was to undertake the first detailed ultramicroscopic analysis of the neodermal surface and attachment structures of Paradiplozoon homoion Bychowsky \& Nagibina, 1959, a generalist parasite infecting a range of cyprinid fish species, including roach Rutillus rutilus (L.), bleak Alburnus alburnus (L.) and gudgeon Gobio gobio Fleming [33, 34]. The species is an oviparous monogenean parasite with a relatively high prevalence in European populations of wild and farmed fish. Our paper forms part of a complex study focusing on diplozoid morphology and ultrastructure and phylogenetic and functional indicators for their specialized life strategy.

\section{Methods}

\section{Sample origin and collection}

Adult $P$. homoion were collected from the gills of bleak caught in the littoral zone of the Mušov lowland reservoir (Czech Republic; 48 53'12"N, 16³4'37"E) in 2013. The fish were transported live in oxygenated water to the parasitological laboratory at the Faculty of Science, Masaryk University, where they underwent a standard parasitological autopsy [35] modified for the detection of diplozoid species, i.e. only the gills were examined. After euthanasia, the gills were extracted and checked for the presence of all diplozoid ontogenetic stages under an
Olympus SZX 7 zoom stereo microscope. Live worms were determined based on their morphology examined under an Olympus BX50 light microscope equipped with Nomarski differential interference contrast and an Olympus Stream Motion digital image analysis system v. 1.9.2. The parasites were then processed and fixed for further light and transmission electron microscopy analysis.

\section{Transmission electron microscopy}

Parasites intended for transmission electron microscopy (TEM) were washed three times in freshwater to remove any remaining mucus. Live specimens were fixed directly in $2 \%$ osmium tetroxide for $1 \mathrm{~h}$ and dehydrated through an ascending acetone series. The dehydrated samples were immediately embedded in Spurr resin [36]. In order to obtain a better orientation in basic diplozoon morphology, ten longitudinal and ten transversal semithin sections $(0.5 \mu \mathrm{m})$ of whole worm bodies were cut (from the anterior extremity of body through the pharynx to the posterior extremity) using a Leica EM UC6i ultramicrotome. Sections were stained with toluidine blue for $30 \mathrm{~s}$ at $80{ }^{\circ} \mathrm{C}$ to contrast the acidic components (sulfates, carboxylates and phosphate radicals) of the different tissues [37]. Selected ultrathin sections were contrasted with uranyl acetate lead by citrate and examined using a JEOL JEM-1010 TEM operating at $60 \mathrm{kV}$. Images were taken for further analysis using Megaview II software (ResAlta Research Technologies).

\section{Results}

\section{General morphological characterization}

The body of $P$. homoion is covered with a neodermal syncytium consisting of multinucleated tissue with no distinct cell boundaries. The hindbody surface is distinctly ridged, with obvious transverse $15 \times 8 \mu \mathrm{m}$ annular ridges located especially in the space between the crossbody and the clamps (Fig. 1a-d). Each of the ridges is supported by muscle fibers lying under the basal lamina and is equipped with scales on the surface plasma membrane (Fig. 1c). The apical part of the ridge inner space, proximal to the basal lamina, is filled with numerous clusters of oval mitochondria with well-developed cristae (Fig. 1b-d). The haptoral clamps, comprising sclerotized elements (sclerites) bounded by muscle tissue, are located on the basal part of the diplozoon hindbody (Fig. 5a-f).

\section{Neodermis}

The $P$. homoion neodermis (tegument) comprises two main parts, an outer syncytial layer located distally to the basal lamina and an inner layer containing the nucleated bodies of the neodermal cells. The inner bodies are located proximally to the basal lamina below the body 

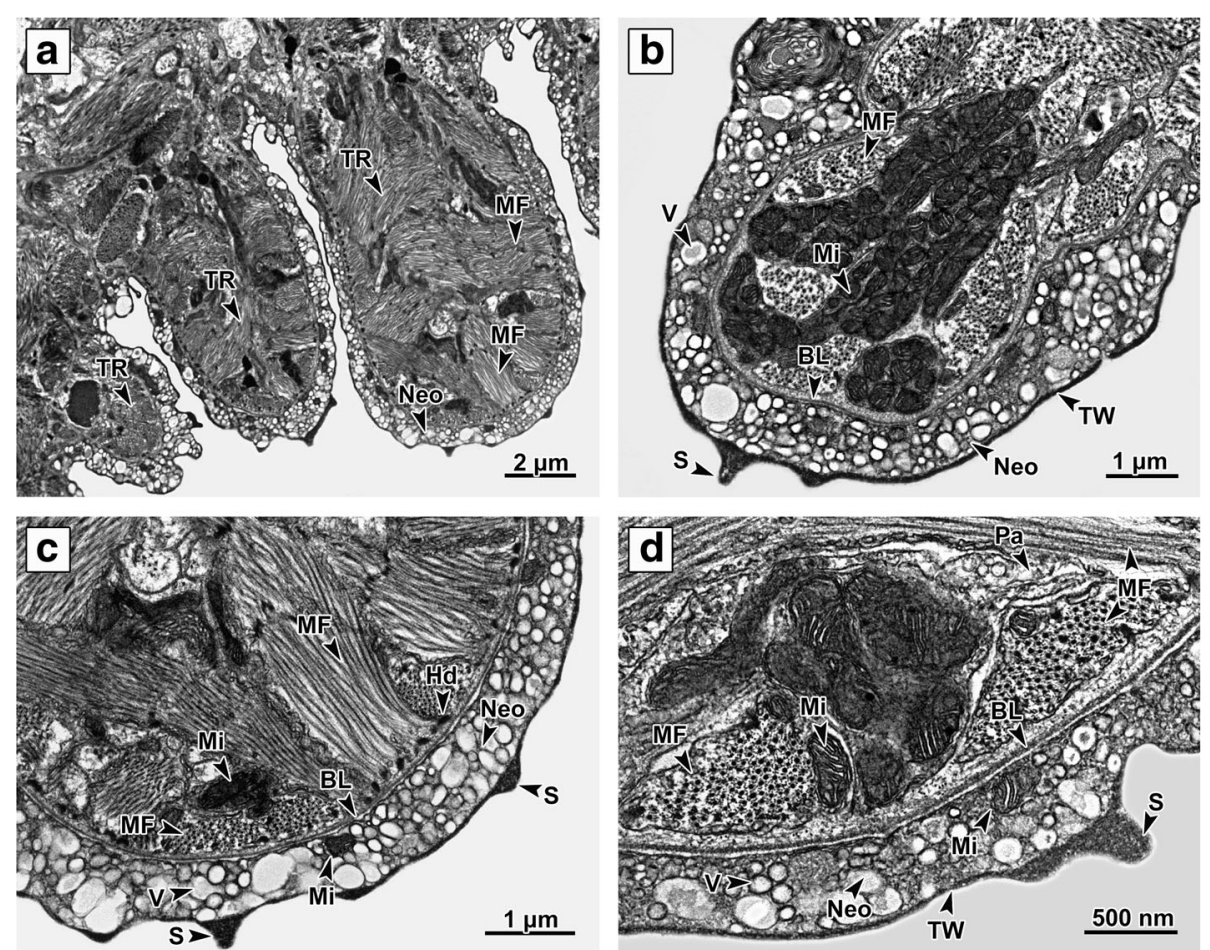

Fig. 1 Body surface ridges. a Longitudinal section through the body surface transverse ridges in the cross-body area. The transverse ridges are supported by numerous multi-directional muscle fibers. b Detailed view through a ridge. The surface of each ridge is covered by a syncytial layer containing numerous vesicles. Scales are located on the external neodermis plasma membrane. Beneath the membrane, the cytoplasm is more electron-dense and fibrous, forming a terminal web. Numerous muscle fibers and mitochondria are located under the basal lamina. c Muscle fibers, attached to the basal lamina by the hemidesmosomes. Note the muscle fibers in different directions. $\mathbf{d}$ Detailed view of the apical part of the ridge. Note (i) the space between the muscle fibers is filled with parenchyma; (ii) the presence of numerous mitochondria with well-developed cristae; (iii) the outer syncytial layer contains different vesicle types and mitochondria; and (iv) the scale connected to the distal plasma membrane. Abbreviations: BL, basal lamina; Hd, hemidesmosomes; Mi, mitochondria; MF, muscle fibers; Neo, neodermis; Pa, parenchyma; S, scales; TR, transverse ridges; TW, terminal web; $V$, neodermal vesicles

wall musculature (Fig. 2a, b). The inner bodies are irregularly shaped and contain large nuclei with a nucleolus, the cytoplasm containing vesicles and scattered mitochondria. The nucleated bodies are connected to the outer layer via cytoplasmic bridges perpendicular to the body surface. The inner bodies and their cytoplasmic bridges were rather scarce and not straight; hence, it was not possible to obtain a complete longitudinal section (Fig. 2a, b). The outer syncytial layer is delimited by the surface and basal plasma membranes and is mainly filled
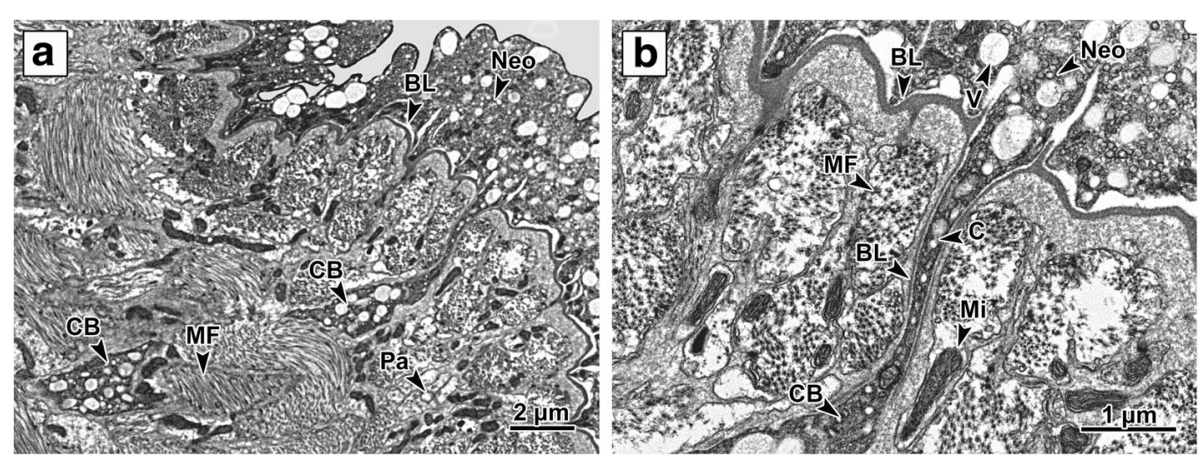

Fig. 2 Cytoplasmic bridge. a The outer syncytial layer located externally to the basal lamina covers whole diplozoon body. The inner nucleated bodies and outer syncytial layer are interconnected by the cytoplasmic bridge. $\mathbf{b}$ Cytoplasmic bridge in detail. The bridge is delimited by the basal lamina and filled with cytoplasm. Numerous muscle fibers and mitochondria are located proximally to the basal lamina. The cytoplasm of external syncytial layer is filled mainly by numerous different granules. Abbreviations: BL, basal lamina; C, cytoplasm; CB, cytoplasmic bridge; MF, muscle fibers; Neo, neodermis; Pa, parenchyma; $V$, neodermal vesicles 
with different types of inclusions. The surface plasma membrane of the neodermal syncytium is covered with external scales, mainly on the hindbody ridges (Fig. 1b). Immediately beneath this membrane, the cytoplasm is more electron-dense and fibrous, forming a region that can be interpreted as a terminal web. The syncytial cytoplasm contains three types of vesicle, a large spherical vesicle (800 $\mathrm{nm}$ in diameter) with fibrous, moderately electron-dense to translucent contents (V1), a small spherical vesicle (250 $\mathrm{nm}$ in diameter) with homogeneous electron-translucent contents (V2), and a very small spherical vesicle $(100 \mathrm{~nm}$ in diameter) with electron-opaque homogeneous contents (V3) (Fig. 3a, b). All vesicle types were located in both the cytoplasmic bridge space and the external layer (Fig. 2a, b). The clusters found in the outer syncytial layer space comprise rounded mitochondria with few cristae surrounded by the outer mitochondrial membrane (Fig. 3a). The basal lamina, consisting of two layers (lamina densa and lamina lucida), lies immediately beneath the basal plasma membrane and has a uniform thickness and numerous deep folds (Figs. 2a, 3b).

The musculature lying proximally to the basal lamina is well developed and formed by several layers of longitudinal and circular muscle fibers, with the circular muscle fibers placed proximally to the basal lamina (Fig. 3b). All muscle fibers are attached to the internal side of the basal lamina by hemidesmosomes (Figs. 1c; 5d, f). The space between the muscle fibers is filled with interstitial material and the fibers themselves are bound by sarcolemma.

The outer syncytial layer covering the anterior forebody is usually of medium width (diameter $3-4 \mu \mathrm{m}$ ), while that covering the apical part of the mouth cavity is 3-7 $\mu \mathrm{m}$ in diameter and equipped with microvilli on its external surface (Figs. 3c, d and 4a, b). The microvilli are covered by plasma membrane and have a dense bundle which serves as its structural core. The surface on the middle forebody is similar to that at the anterior forebody, being of medium-width (c.4 $\mu \mathrm{m}$ diameter) and having a smooth, slightly undulating surface (Fig. 4c). The cross-body area is covered with a thick $(c .6 \mu \mathrm{m}$ wide) outer layer with an undulating surface (Fig. 4d). This is the thickest part of the P. homoion surface external layer. The middle hindbody external layer is ridged and is covered with a thin, smooth outer syncytial layer with maximum width of $1-2 \mu \mathrm{m}$ with a few scales on the external side (Fig. 4e). The outer syncytial layer covering the clamps is regular, smooth on its surface and
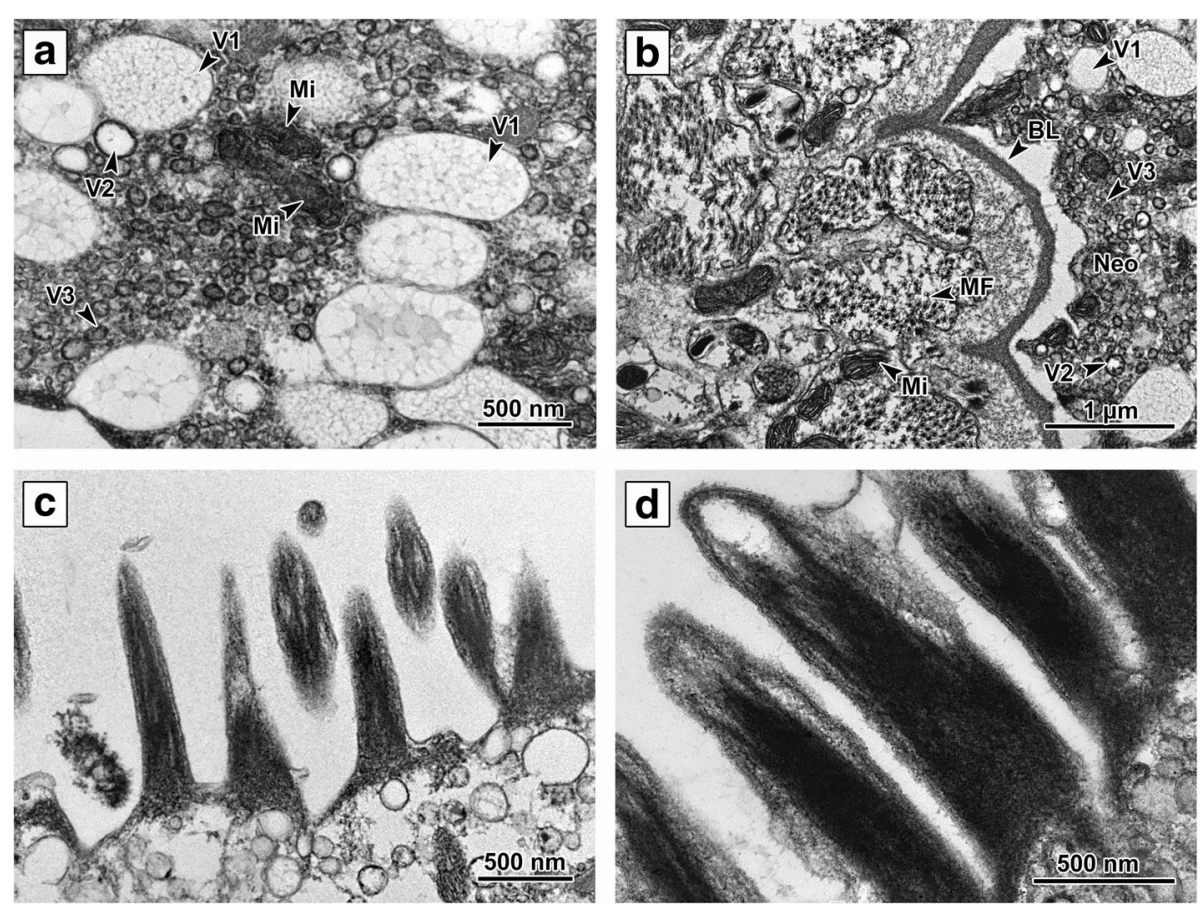

Fig. 3 Ultrastructure of the outer layer. a The cytoplasm of the outer syncytial layer is filled with different vesicle types. Small groups of mitochondria with few cristae are scattered between the vesicles in the outer syncytial layer space. b The outer syncytial layer, with vesicles, on the distal side of the basal lamina. Several layers of circular and longitudinal muscle fibers are located proximally to the basal lamina. Basal lamina protuberances directing proximally to the subsurface area; the protuberances denoting the presence of cytoplasmic bridges connecting the nucleated cell bodies and the external syncytial layer. c External surface of mouth cavity neodermal syncytial layer covered with microvilli. $\mathbf{d}$ Detail of the microvilli; these are covered by plasma membrane and have a dense bundle which serves as a structural core. Abbreviations: BL, basal lamina; Mi, mitochondria; MF, muscle fibers; Neo, neodermis; V1, V2, V3, vesicle types 

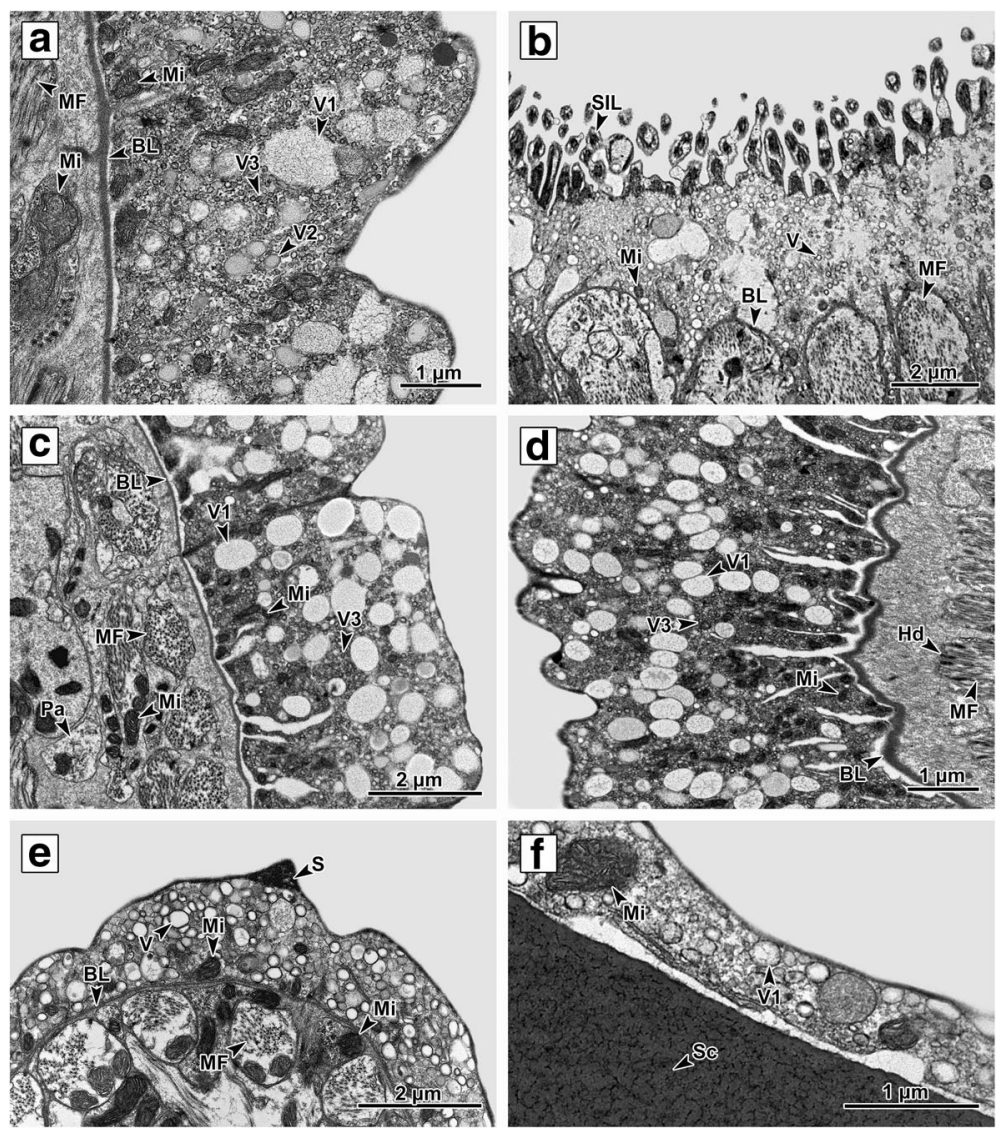

Fig. 4 Variation in the outer layer from different body parts. a Anterior part of the forebody. b Apical part of the mouth cavity; note the layer with microvilli directing toward the mouth cavity lumen on the external surface. c Middle part of the forebody. $\mathbf{d}$ Cross-body area. The syncytial cytoplasm is filled with numerous mitochondria and a considerable number of $\mathrm{V} 1$ vesicles. e Middle part of the hindbody ridged with a few scales located on the distal surface. f Clamp outer syncytial layer. Abbreviations: BL, basal lamina; Hd, hemidesmosomes; Mi, mitochondria; MF, muscle fibers; Neo, neodermis; Pa, parenchyma; V1, V2, V3, vesicle types; S, scale; Sc, sclerite; SIL, microvilli; V, neodermal vesicles

very thin, with a maximum width of $0.75 \mu \mathrm{m}$. This is the thinnest part of the external surface layer (Fig. 4f). Large mitochondria (500 $\mu \mathrm{m}$ in diameter) were detected in the cytoplasm of the clamp outer layer. The outer syncytial layer on the middle forebody and cross-body region contains a higher proportion of V1 vesicles (Fig. 4c, d). This is in contrast to the other body parts, where the V2 and $\mathrm{V} 3$ vesicles tend to predominate (Fig. 4a, b, e, f).

\section{Clamps}

Each of the clamps has a flattened bowl-like shape, the structure comprising sclerites and movable skeletal-like structures anchored by robust, radially oriented muscle bundles (Fig. 5a-f). The clamps are covered by a thin neodermal syncytial layer over the whole surface. This layer is thinner than that covering the rest of the body (Fig. 6c, d).

Each sclerite is closely bound by the basal lamina of the surrounding muscle complex (Fig. 6b). Between the body of the sclerites and the surrounding basal lamina there is a space filled with closely packed fibrous content known as the outer layer (Fig. 6b). The sclerite body is composed of a moderately electron-dense material containing fibrils, which are irregularly organized and of different lengths; many of them are branched, forming randomly orientated three-dimensional fibrous structures (Fig. 6a, b). Large cavities with closely packed fibrils, mitochondria and numerous small electron-dense granules inside the lumen are clearly visible in longitudinal sections through the sclerites (Fig. 6a). Nerve fibers were located relatively close to the terminal and basal parts of sclerites (Figs. 5a, b, d and 6c, d). Very small (0.1 $\mu \mathrm{m}$ in diameter) electron-dense, membrane-bound vesicles were detected adhering to the external surface of the sclerites (Fig. 5c). Some vesicles were also located in the parenchyma space and inside sclerites on the internal surface of the cavities.

The base of the posterior median plate sclerites is equipped with glandular cells possessing secretory vesicles (Fig. 5e). The same secretory vesicles are located in 

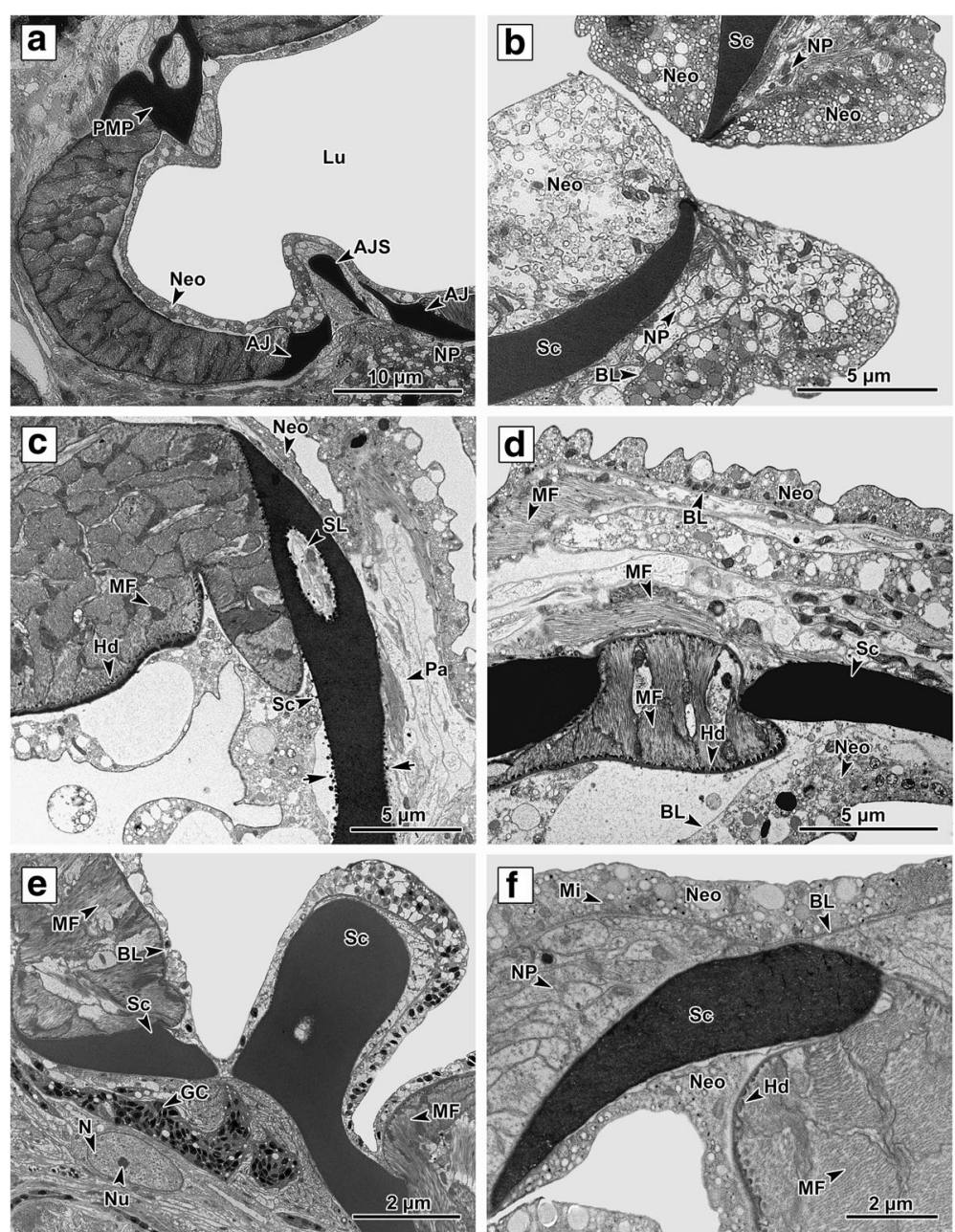

Fig. 5 Ultrastructure of clamp I. a The central part of two anterior jaw sclerites connected by an anterior joining sclerite and the posterior part of a median plate sclerite. The nerve fibres are located at the base of the anterior joining sclerite. $\mathbf{b}$ Terminal part of both jaws forming the clamp, i.e. the part coming in direct contact with host tissue (gills). c A cross-section of the clamp musculature, with hemidesmosomes connected to the lateral sclerite; note also the electron-dense material (arrows) adherent sclerite surface. $\mathbf{d}$ A detail of the sclerite surrounded by radial muscle fibers with clearly visible hemidesmosomes. e A detail of the posterior median plate sclerite; note the glandular cell with secretory vesicles and a nucleus with nucleolus in the cytoplasm at the basal area of the sclerite. Secretory vesicles are spread throughout the syncytial layer cytoplasm. $\mathbf{f}$ A detail of the anterior jaw sclerite, showing the large nerve plexus proximally to the external syncytial layer and the well-developed musculature, enabling clamp movement. Abbreviations: AJ, anterior jaw sclerites; AJS, anterior joining sclerite; BL, basal lamina; GC, glandular cell; Hd, hemidesmosomes; Lu, luminal part of the clamp; MF, muscle fibers; N, nucleus; Neo, Neodermis; Nu, nucleolus; NP, nerve fiber; PMP, posterior part of median plate; Sc, sclerite; SL, sclerite lumen

the surface external syncytial layer covering the medial plate sclerite.

\section{Discussion}

\section{General morphological characterization}

Comparison with previous studies concerning platyhelminth surface structures [38-40] confirms that $P$. homoion share analogous morphological characteristics with other Platyhelminthes. While there have been numerous previous studies focused on the morphology and ultrastructure of monogenean surface and attachment structures using TEM [10, 11, 23, 24, 26, 27, 41], none have included species from the family Diplozoidae. Highly developed transverse tegumentary annular ridges were identified on the hindbody of Eudiplozoon nipponicum using scanning electron microscopy [42]. The same ridges were later recognized on histological sections and termed "tegumental folds" [43]. Our findings on $P$. homoion suggest that such ridges are not solely of outer neodermal (tegumental) syncytium origin, the ridges comprising well-developed musculature, the surface of which is covered with an outer syncytial layer. The outer surface layer of the hindbody ridges is equipped with several electron-dense scales on the external plasmatic membrane. Epidermal scales were also recorded on the posterior surface of the monogenean $D$. aequans [9]. 

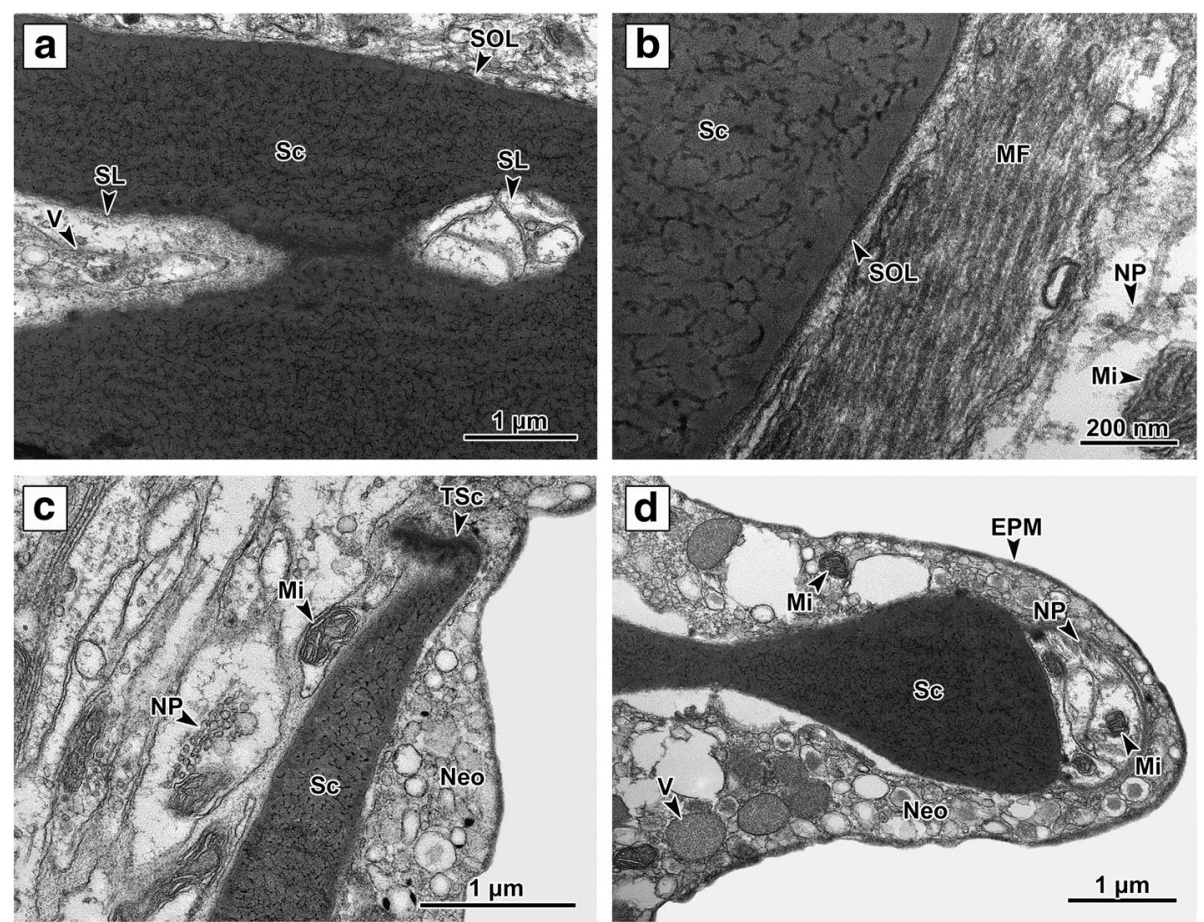

Fig. 6 Ultrastructure of clamp II. a A clamp sclerite limited distally by the outer layer, with a sclerite lumen inside. b A section through the muscle fibers enabling sclerite movement. The muscle fibers are attached to the sclerite on its outer layer. c Terminal part of the sclerite with its hooked tip. $\mathbf{d}$ Detail of the terminal part of the anterior joining sclerite. A large nerve fiber is located on the terminal part of the sclerite. Abbreviations: EPM, external plasma membrane; Mi, mitochondria; MF, muscle fibers; Neo, neodermis; NP, nerve fiber; Sc, sclerite; SL, sclerite lumen; SOL, outer layer; TSc, terminal part of the sclerite; $V$, vesicles

These scales, which were composed of moderately electron-dense material, were located within the tegumental external syncytial layer cytoplasm, beneath the outer membrane. Relatively large and apparently hard scale-like neodermal structures have been reported as 'spines' or 'scales' in the monogenean Pseudorhabdosynochus spp. (Diplectanidea) [44], but these were not examined at an ultrastructural level. While the function of the scales on $P$. homoion is still not fully understood, their presence could suggest a protective role.

\section{Neodermis}

The primary surface structure of $P$. homoion is very similar to that of other monogenean species, e.g. D. merlangi [7]; R. emarginata, P. gurnardi [8]; Pricea multae [25]; Atriaster sp. [45]; Tetraonchoides sp. [46]; M. macracantha [11]; Dictyocotyle coeliaca [47]. In each of these, the outer syncytial layer is connected to the nucleated cell body via cytoplasmic bridges.

Different granular and vesicular bodies have been observed in the syncytial layer cytoplasm in all previously studied monogeneans [12, 26, 45, 48, 49]. These bodies are produced by Golgi stacks in the inner nucleated regions and exported to the outer syncytial layer. This corresponds well with our TEM results for $P$. homoion, whereby the vesicles were present in both inner bodies and the outer layer. Unlike Gotocotyla bivaginalis [26], for which four vesicle types were documented in the outer cytoplasm layer, we observed only three types in $P$. homoion.

Previous studies have noted differences in the surface ultrastructure of two heteronchoinean monogeneans $(R$. emarginata and P. gurnardi) [8]. Uniquely among monogeneans, for example, $R$. emarginata possess a series of shallow pore-like infoldings at their free edge, and lack microvilli. The surface of the diclidophorid $P$. gurnardi, on the other hand, is similar to that of other monogeneans. The surface structure of $P$. homoion differs from both of these species in that no shallow pore-like infoldings or microvilli were recorded anywhere on the surface (except around the mouth cavity). Finally, while the apical membranes of Atriaster sp. [45] have some undulations and microvilli, similar to E. soleae or A. elegans [6], they are lacking in both $R$. emarginata [8] and P. homoion.

While the $P$. homoion neodermis possesses the same morphological properties in different body regions, slight differences could be discerned in the width of the outer syncytial layer and the amount of individual organelles. The outer syncytial layer on the middle forebody and cross-body region of $P$. homoion, for example, contains a higher proportion of $\mathrm{V} 1$ vesicles, in contrast to other 
body parts where V2 and V3 vesicles tend to predominate. Although the meaning of the vesicles has not yet been clarified in Diplozoon spp., these may have a storage function. The neodermal organelles and the role of the neodermis (tegument) in nutrient uptake by parasitic platyhelminths have previously been discussed by Dalton et al. [50]. In $P$. homoion, the outer syncytial layer around the apical mouth cavity, with its microvilli, ranging between 3 and $7 \mu \mathrm{m}$ in total length. The different measurements recorded could be related to the parasite's feeding technique, the mouth cavity having to be very flexible while sucking fish blood. Although the microvilli on the surface neodermis of the mouth have been discussed previously [14], their true function is still far from being understood, though it is likely to be related either to disruption of host tissue or uptake of host blood.

The clamps of $P$. homoion are covered with a very thin outer syncytial layer, which correlates well with results obtained for other monogenean species, such as G. bivaginalis and C. leptogaster [26, 27]. This reduction in external syncytial layer thickness probably reflects an adaptation related to the parasite's life strategy, in that the clamps must be able to grab hold of tissue between the host's gill lamellae in a very limited space.

\section{Clamps}

While morphology and sclerite composition has frequently been studied in relation to species identification, morphological abnormalities, and taxonomic characterization of species of the family Diplozoidae [22, 51-53], there have been no ultrastructural studies on the clamps. Our findings on $P$. homoion correlate with observations on the clamps of other heteronchoinean monogeneans in that the clamp frame is usually formed of several electron-dense sclerites bound by radial muscle fibers, the surface is covered with a very thin outer syncytial layer, and nerve fibers are located close to the terminal and basal parts of the sclerites [26-28]. Association of nerve plexuses with the clamps has also been observed in E. nipponicum (a species closely related to $P$. homoion) using immunostaining and microscopic observation [54].

Early studies suggested that Paradiplozoon sp. sclerites were composed of chitin [55]; however, later work showed that the sclerites of $D$. paradoxum from the same family consisted of a resilin-like protein [32]. More recently, studies on the chemical composition of monogenean clamps have shown that they are formed of scleroproteins, probably stabilized by tyrosyl residues in dityrosine [29, 56].

Studies on the development and ultrastructure of Gastrocotyle trachuri clamp walls have detected numerous differentiating cells associated with the region around the epidermal infoldings and developing clamp $[24,57]$. These cells produce cytoplasmic extensions that form a syncytial region around the epidermal infolding near the region of clamp formation. The same cells also produce groups of electron-lucent membrane-bound vesicles that pass along the extensions towards the region of clamp formation. In a previous study describing the mechanism of sclerite development [57], the authors noted formation of short hollow tubules at the start of the process, followed by an increasing number of tubules and development of areas of dense material within the developing sclerites, after which the sclerites became uniformly electron-dense prior to forming the clamps. We recorded electron-dense material migrating through the parenchyma to the sclerite surface, an observation supported by previous observations on G. trachuri [57], in which electron-lucent membrane-bound vesicles were observed moving along the differentiating cell extensions towards the region of clamp formation.

Here, we document for the first time, glandular cells containing secretory bodies at the base of the posterior median plate sclerite. Numerous gland cells have been observed opening ventrally into the ducts around the haptor suckers in Polystomoides spp. [58]. Cyton-containing secretory bodies of different sizes and shapes have been described between numerous membranous folds near the marginal hooklets in Macrogyrodactylus congolensis [59], although it is not thought that the glandular secretions play any role in attachment to the host's skin by the marginal hooklets. The function of the haptoral gland cells at the base of the median plate sclerite, observed for the first time in our study, is still not fully understood and merits further attention. However, as three types of secretory products related to attachment have been observed in the anterior adhesive areas of two capsalid Benedenia monogeneans (B. rohdei and B. lutjani) [60], they could be associated with the process of attachment to the fish gills.

\section{Conclusion}

Our work represents a first approach for examination the surface and attachments clamp ultrastructure in a species of the family Diplozoidae using TEM. Ultrastructural details were characterized for a range of tegumental compartments, including scales located on the hindbody, the outer surface layer, and the cytoplasmic bridge connecting the inner nucleated cell bodies and the outer syncytial layer. Our findings for $P$. homoion contradict previous information that the surface ridges are purely of neodermal origin; rather, the ridges show well-developed musculature and a neodermal outer layer localized externally to the basal lamina that covers the ridge's surface. This study provides a unique comparison of the neodermis from different body parts for species from the family Diplozoidae. We were able to produce unique photo documentation of electron-dense material (responsible for sclerite development) migrating through 
the parenchyma to the sclerite surface. Similarly, we also documented the occurrence of glandular cells at the base of attachment clamps for the first time in a diplozoid species. The mechanisms involved in sclerite and glandular cell development merit further attention.

\section{Acknowledgements}

The authors would like to thank the Faculty of Science, Masaryk University Brno. A big thank you goes to Professor Iva Dyková for her help with TEM observations, her helpful comments and kind approach. We acknowledge the Laboratory of EM, Biology Centre of CAS, institution supported by the MEYS CR (LM 2015062 Czech - Biolmaging). Many thanks go to Gabriela Vágnerová and Eliška Šrámová for their help with fish dissections and to Dr. Kevin Roche for English correction of this text.

\section{Funding}

The study, VK, BK and MG were supported by Czech Science Foundation Grant No. GAP506/12/1258. MK and JI were supported by the Czech Science Foundation (GBP505/12/G112-ECIP). VK and Jl also received financial support via the Grant Agency of Zasaryk University, Category A, No. MUNI/A/1325/2015.

\section{Availability of data and materials}

The data supporting the conclusions of this article are included within the article.

\section{Authors' contributions}

VK, BK and MG conceived and designed the study. MG provided scientific background in the field of monogenean research. VK, BK, MK and Jl provided the dissections. VK conducted the TEM analysis. JI designed the tables. MK revised the manuscript critically for important intellectual content. VK analyzed data and wrote the paper. All authors contributed to improve the manuscript and read and approved the final version.

\section{Competing interests}

The authors declare that they have no competing interests.

\section{Consent for publication}

Not applicable.

\section{Ethics approval and consent to participate}

Not applicable.

\section{Publisher's Note}

Springer Nature remains neutral with regard to jurisdictional claims in published maps and institutional affiliations.

\section{Author details}

'Department of Botany and Zoology, Faculty of Science, Masaryk University, Kotlárská 2, 61137 Brno, Czech Republic. ${ }^{2}$ Department of Parasitology, Faculty of Science, Charles University, Viničná 7, 12844 Prague, Czech Republic. ${ }^{3}$ Department of Medical Biology, Faculty of Medical Sciences, University of Warmia and Mazury in Olsztyn, Żolnierska 14c, 10-561 Olsztyn, Poland.

Received: 7 March 2017 Accepted: 17 May 2017

Published online: 25 May 2017

\section{References}

1. Kawatsu H. Studies on the anemia of fish - IX. Hypochromic microcytic anemia of crucian carp caused by infestation with a trematode, Diplozoon nipponicum. Bull Japan Soc Sci Fish. 1978;44:1315-9.

2. Buchmann K, Bresciani J. Monogenea (Phylum Platyhelminthes). Fish Dis Disorders. 2006:1:297-344.

3. Khotenovsky IA. Life cycle of several monogeneans of the genus Diplozoon. Parazitol Sb. 1977:27:35-43. (In Russian)

4. Lyons KM. Fine structure of the outer epidermis of the viviparous monogenean Gyrodactylus sp. from the skin of Gasterosteus aculeatus. J Parasitol. 1970;56:1110-7.
5. Kritsky DC, Kruidenier FJ. Fine structure and development of the body wall in the monogenean, Gyrodactylus eucaliae Ikezaki and Hoffman, 1957. Proc Helminthol Soc Wash. 1976;43:47-58.

6. Lyons $\mathrm{KM}$. The fine structure and function of the adult epidermis of two skin parasitic monogeneans, Entobdella soleae and Acanthocotyle elegans. Parasitology. 1970;60:39-52.

7. Morris GP, Halton DW. Electron microscope studies of Diclidophora merlangi (Monogenea: Polyopisthocotylea). II. Ultrastructure of the tegument. J Parasitol. 1971;57:49-61.

8. Lyons KM. Ultrastructural observations on the epidermis of the polyopisthocotylinean monogeneans Rajonchocotyle emarginata and Plectanocotyle gurnardi. Z Parasitenkd. 1972;40:87-100.

9. Shaw MK. The ultrastructure of the epidermis of Diplectanum aequans (Monogenea). Parasitology. 1980;80:9-21.

10. Ramasamy P, Brennan GP, Halton DW. Ultrastructure of the surface structures of Allodiscocotyla diacanthi (Polyopisthocotylea: Monogenea) from the gills of the marine teleost fish, Scomberoides tol. Int J Parasitol. 1995;25:43-54

11. Cohen SC, Kohn A, Baptista-Farias MFD. Ultrastructure of the tegument of Metamicrocotyla macracantha (Alexander, 1954) Koratha, 1955 (Monogenea, Microcotylidae). Braz J Biol. 2004;64:27-31.

12. Smyth JD, Halton DW. The physiology of trematodes. Cambridge: Cambridge University Press; 1983.

13. Tyler S, Hooge M. Comparative morphology of the body wall in flatworms (Platyhelminthes). Can J Zool. 2004;82:194-210.

14. Konstanzová V, Koubková B, Kašný M, llgová J, Dzika E, Gelnar M. Ultrastructure of the digestive tract of Paradiplozoon homoion (Monogenea). Parasitol Res. 2015:114:1485-94.

15. Llewellyn J. The adhesive mechanisms of monogenetic trematodes: the attachment of Plectanocotyle gurnardi (v. Ben. \& Hesse) to the gills of Trigla. J Mar Biol Assoc UK. 1956;35:507-14.

16. Llewellyn J, Owen IL. The attachment of the monogenean Discocotyle sagittata Leuckart to the gills of Salmo trutta L. Parasitology. 1960;50:51-60.

17. Owen IL. The attachment of the monogenean Diplozoon paradoxum to the gills of Rutilus rutilus LI Micro-habitat and adhesive attitude. Parasitology. 1963:53:455-61.

18. Khotenovsky IA. Fauna of the USSR. Monogenea. Suborder Octomacrinae Khotenovsky. Leningrad: Nauka; 1985. (In Russian).

19. Pečínková M, Vøllestad LA, Koubková B, Gelnar M. Asymmetries in the attachment apparatus of a gill parasite. J Zool. 2007;272:406-14.

20. Bychowsky BE. Monogenetic trematodes, their classification and phylogeny. Moscow - Leningrad: Academy of Sciences USSR; 1957. (In Russian).

21. Bychowsky BE, Nagibina LF. On the systematics of the genus Diplozoon Nordmann (Monogenoidea). Zool Zh. 1959;38:362-77. (In Russian).

22. Šebelová Š, Kuperman B, Gelnar M. Abnormalities of the attachment clamps of representatives of the family Diplozoidae. J Helminthol. 2002;76:249-68.

23. Shaw MK. The ultrastructure of the clamp sclerites in Gastrocotyle trachuri and other clamp-bearing monogeneans. Z Parasitenkd. 1979;59:43-51.

24. Shaw MK. The ultrastructure of the clamp wall of the monogenean gill parasite Gastrocotyle trachuri. Z Parasitenkd. 1979;58:243-58.

25. Ramasamy P, Hanna REB, Threadgold T. The surface topography and ultrastructure of the tegument and haptor of Pricea multae (Monogenea). Int J Parasitol. 1986:16:581-9.

26. Ramasamy P, Bhuvaneswari R. The ultrastructure of the tegument and clamp attachment organ of Gotocotyla bivaginalis (Monogenea, Polyopisthocotylea). Int J Parasitol. 1993;23:213-20.

27. Poddubnaya LG, Hemmingsen W, Gibson DI. Clamp ultrastructure of the basal monogenean Chimaericola leptogaster (Leuckart, 1830) (Polyopisthocotylea: Chimaericolidae). Parasitol Res. 2014;113:4023-32.

28. Poddubnaya LG, Hemmingsen W, Gibson DI. Ultrastructural observations of the attachment organs of the monogenean Rajonchocotyle emarginata (Olsson, 1876) (Polyopisthocotylea: Hexabothriidae), a gill parasite of rays. Parasitol Res. 2016;115:2285-97.

29. Lyons KM. The chemical nature and evolutionary significance of monogenean attachment sclerites. Parasitology. 1966;56:63-101.

30. Kayton RJ. Histochemical and X-ray elemental analysis of the sclerites of Gyrodactylus spp. (Platyhelminthes: Monogenoidea) from the Utah chub, Gila atraria (Girard). J Parasitol. 1983;69:862-5.

31. Shinn AP, Gibson DI, Sommerville C. A study of the composition of the sclerites of Gyrodactylus Nordmann, 1832 (Monogenea) using X-ray elemental analysis. Int J Parasitol. 1985;25:797-805. 
32. Wong WL, Michels J, Gorb SN. Resilin-like protein in the clamp sclerites of the gill monogenean Diplozoon paradoxum Nordmann, 1832. Parasitology. 2013;140:95-8.

33. Matejusová I, Koubková B, Gelnar M, Cunningham CO. Paradiplozoon homoion Bychowsky \& Nagibina, 1959 versus P. gracile Reichenbach-Klinke, 1961 (Monogenea): two species or phenotypic plasticity? Syst Parasitol. 2002;53:39-47

34. Košková E, Špakulová M, Koubková B, Reblánová M, Orosová M. Comparative karyological analysis of four diplozoid species (Monogenea, Diplozoidae), gill parasites of cyprinid fishes. Parasitol Res. 2011;108:935-41.

35. Ergens R, Lom J. Causative agents of parasitic diseases of fish. Prague: Academia; 1970.

36. Spurr AR. A low-viscosity epoxy resin embedding medium for electron microscopy. J Ultra Res. 1969;26:31-43.

37. Trump BF, Smuckler EA, Benditt EP. A method for staining epoxy sections for light microscopy. J Ultra Res. 1961;5:343-8.

38. Morseth DJ. The fine structure of the tegument of adult Echinococcus granulosus, Taenia hydatigena, and Taenia pisiformis. J Parasitol. 1966;52: 1074-85.

39. Morris GP, Threadgold LT. Ultrastructure of the tegument of adult Schistosoma mansoni. J Parasitol. 1968;54:15-27.

40. Filippi JJ, Quilichini Y, Foata J, Marchand B. Topography and ultrastructure of the tegument of Lecithochirium musculus (Digenea: Hemiuridae), a parasite of the European eel Anguilla anguilla (Osteichthyes: Anguillidae). J Morphol. 2012;273:361-70.

41. Santos CP, Lanfredi RM. Ultrastructure and cytochemistry of the tegument of Atriaster heterodus (Platyhelminthes: Monogenea). Mem Inst Oswaldo Cruz. 2000;95:899-904.

42. Hodová I, Matejusova I, Gelnar M. The surface topography of Eudiplozoon nipponicum (Monogenea) developmental stages parasitizing carp (Cyprinus carpio L.). Cent Eur J Biol. 2010;5:702-9.

43. Valigurová A, Hodová I, Sonnek R, Koubková B, Gelnar M. Eudiplozoon nipponicum in focus: monogenean exhibiting a highly specialized adaptation for ectoparasitic lifestyle. Parasitol Res. 2011;108:383-94.

44. Santos CP, Buchmann K, Gibson DI. Pseudorhabdosynochus spp. (Monogenea: Diplectanidae) from the gills of Epinephelus spp. in Brazilian waters. Syst Parasitol. 2000;45:145-53.

45. Justine $\mathrm{J}$. Ultrastructure of spermiogenesis, spermatozoa and the tegumen in Atriaster sp. (Platyhelminthes, Monogenea, Polyopisthocotylea, Microcotylidae). Zool Scr. 1992;21:231-8.

46. Justine JL, Mattei X, Euzet L. Ultrastructure of Tetraonchoides (Platyhelminthes, Monogenea): tegument, tegumentary receptors, oocytes and mineralous corpuscles. Ann Sci Nat Zool Biol Anim. 1994;15:151-62.

47. Poddubnaya LG, Hemmingsen W, Gibson DI. Surface ultrastructural characteristics of Dictyocotyle coeliaca Nybelin, 1941 (Monopisthocotylea: Monocotylidae), an endoparasitic monogenean of rays. Parasitol Res. 2016; 115:965-73.

48. El-Naggar MM, Kearn GC. The tegument of the monogenean gill parasites Dactylogyrus amphibothrium and D. hemiamphibothrium. Int J Parasitol. 1983;13:579-92.

49. Ramasamy P, Brennan GP. Ultrastructure of the surface structures and haptor of Empleurosoma pyriforme (Ancyrocephalinae; Monopisthocotylea: Monogenea) from the gills of the teleost fish Therapon jarbua. Parasitol Res. 2000;86:129-39.

50. Dalton JP, Skelly P, Halton DW. Role of the tegument and gut in nutrient uptake by parasitic platyhelminths. Can J Zool. 2004;82:211-32.

51. Civáňová K, Koyun M, Koubková B. The molecular and morphometrical description of a new diplozoid species from the gills of the Garra rufa (Heckel, 1843) (Cyprinidae) from Turkey - including a commentary on taxonomic division of Diplozoidae. Parasitol Res. 2013;112:3053-62.

52. Avenant-Oldewage A, Le Roux LE, Mashego SN, van Vuuren BJ. Paradiplozoon ichthyoxanthon n. sp. (Monogenea: Diplozoidae) from Labeobarbus aeneus (Cyprinidae) in the Vaal River, South Africa. J Helminthol. 2014;88:166-72.

53. Pečínková M, Matejusova I, Koubková B, Gelnar M. Classification and occurrence of abnormally developed Paradiplozoon homoion (Monogenea, Diplozoinae) parasitising gudgeon Gobio gobio. Dis Aquat Org. 2005;64:63-8.

54. Zurawski TH, Mousley A, Mair GR, Brennan GP, Maule AG, Gelnar M, Halton DW. Immunomicroscopical observations on the nervous system of adult Eudiplozoon nipponicum (Monogenea: Diplozoidae). Int J Parasitol. 2001;31: 783-92.
55. Milne SJ, Avenant-Oldewage A. The fluorescent detection of Paradiplozoon sp. (Monogenea: Diplozoidae) attachment clamps' sclerites and integumental proteins. Onderstepoort J Vet Res. 2006;73:149-52.

56. Ramalingam K. Chemical nature of monogenean sclerites. I. Stabilization of clamp-protein by formation of dityrosine. Parasitology. 1973;66:1-7.

57. Shaw MK. The development of the clamp attachment organs of the monogenean Gastrocotyle trachuri. Z Parasitenkd. 1979;59:277-94.

58. Rohde K. Fine structure of the Monogenea, especially Polystomoides Ward. Adv Parasitol. 1975;13:1-33.

59. Arafa S. Ultrastructure of musculature of the marginal hooklets of Macrogyrodactylus congolensis, a monogenean skin parasite from the catfish Clarias gariepinus. Acta Parasit. 2011;56:122-30.

60. Whittington ID, Cribb BW. Morphology and ultrastructure of the anterior adhesive areas of the capsalid monogenean parasites Benedenia rohdei from the gills and B. lutjani from the pelvic fins of Lutjanus carponotatus (Pisces: Lutjanidae). Parasitol Res. 1999;85:399-408.

\section{Submit your next manuscript to BioMed Central and we will help you at every step:}

- We accept pre-submission inquiries

- Our selector tool helps you to find the most relevant journal

- We provide round the clock customer support

- Convenient online submission

- Thorough peer review

- Inclusion in PubMed and all major indexing services

- Maximum visibility for your research

Submit your manuscript at www.biomedcentral.com/submit
) Biomed Central 